\title{
Prevalence and associated factors for suicidal ideation in the Lagos State Mental Health Survey, Nigeria
}

Abiodun O. Adewuya, Bolanle A. Ola, Olurotimi A. Coker, Olayinka Atilola, Mathew P. Zachariah, Olufemi Olugbile, Adedolapo Fasawe and Olajide Idris

\section{Background}

To combat the increasing rate of suicide, basic data on suicidal behaviours reflecting the uniqueness of the locality are needed in sub-Saharan Africa.

\section{Aims}

To assess the prevalence of suicidal ideation and associated factors.

\section{Method}

Adults ( $n=11246$ ) from the five administrative divisions of Lagos State completed questionnaires detailing suicidal ideation, socio-demographic details, common mental disorders (depression, anxiety and somatic symptoms), alcohol and psychoactive substance use disorders and disability.

\section{Results}

The weighted prevalence of current suicidal ideation was 7.28\% (s.e. 0.27). Independently associated factors were older age, being female, not married, low occupational group, depression, anxiety, somatic symptoms and disability.

\section{Conclusions}

Despite the validity of cross-national surveys, there is need for individual countries to generate complementary local data to explain variability in rates and risk factors in order to plan for suicide prevention or develop timely and effective response.

\section{Declaration of interest \\ None.}

\section{Copyright and usage}

(c) The Royal College of Psychiatrists 2016. This is an open access article distributed under the terms of the Creative Commons Non-Commercial, No Derivatives (CC BY-NC-ND) license.
It is estimated that about 800000 people die by suicide every year, representing an annual age-standardised suicide rate of 11.4 per 100000 population globally and 6.11 per 100000 population in Nigeria specifically. ${ }^{1}$ Suicide is one of the three leading causes of death among those in the most economically productive age group (15-44 years). ${ }^{2}$ It is a public health issue that is estimated to contribute more than $2 \%$ to the global burden of disease by the year 2020, especially in the sub-Saharan African countries where services are scarce. ${ }^{3}$ Suicide was recently identified by the World Health Organization (WHO) as a priority condition in the Mental Health Gap Action Programme (mhGAP). ${ }^{4}$ In its 66th World Health Assembly, the WHO adopted the first-ever Mental Health Action Plan with suicide prevention as an integral part of he plan, with the goal of reducing the rate of suicide in countries by $10 \%$ by $2020 .^{5}$ With this major shift in attention towards the prevention of suicide, epidemiological research and basic data on the prevalence and risk factors for suicidal behaviours (ideation, plan and attempt) are urgently needed in many countries of subSaharan Africa.

There had been several cross-national surveys including the NIMH Epidemiological Catchment Area Study, ${ }^{6}$ the National Comorbidity Survey ${ }^{7}$ and, later, the World Mental Health Survey (WMHS), which had estimated the rate of suicidal ideation at 9.2\% globally (3.2\% for Nigeria) and had female, younger age, less educated, unmarried and having a mental disorder as the risk factors. ${ }^{8}$ However, despite the validity and popularity of these surveys, there may be some reservations in adopting their findings for planning for the local environment as we believe that crossnational data may not adequately reflect the specifics and uniqueness of a country or district. Also, the WMHS assessed for lifetime prevalence, which, apart from being prone to serious recall bias, may not have relevance to present feeling of the individual, so cannot be used to determine the population in need of help at a particular time so as to plan prevention services. Specifically, the two-stage sampling design resulting in the small sample size for Nigeria data and the non-evaluation of somatic symptoms (which is common to Africans) as a possible risk factor limits the use of the WMHS findings for service planning in Nigeria. Moreover, the WMHS was conducted over 14 years ago and the trend could have changed since then.

Lagos (population 16 million) ${ }^{9}$ accounts for about $10 \%$ of Nigeria's population and is reputed to be among the top five most densely populated and most stressful cities in the world. Although originally inhabited by the 'Awori group' of the Yoruba people, today Lagos State has a very diverse population because of heavy migration from other parts of Nigeria and surrounding countries. Although the Yoruba are the dominant ethnic group, there are more than 250 ethnic groups represented in Lagos, including the Hausa, Igbo and Fulani. It has been estimated that about $66 \%$ of the population lives in slums. Lagos has been dubbed the 'megacity of slums', with millions living in and around the lagoons with no access to roads, clean water, electricity or waste disposal. The World Bank has identified at least 12 large slums of the city in serious need of intervention. ${ }^{10}$

To generate data for planning mental health services, the Lagos State Mental Health Survey (LSMHS) was initiated to assess the types, patterns and severity of common mental health problems among citizens of Lagos State. The overall aim of this study was to estimate the current prevalence of suicidal ideation and examine the associated factors using the data from the LSMHS. It is hypothesised that the 'current' lifetime prevalence may be higher and more valid than the previously described lifetime prevalence. 


\section{Method}

\section{Sampling}

A mixed method of both cluster and systematic random sampling was used to determine the areas included in the project. This was to minimise potential bias, increase the power to examine the social contexts of mental health problems and make it possible to compare individuals recruited in different geographical areas of the state.

Lagos State is divided into five administrative divisions (Ikeja, Badagry, Ikorodu, Lagos and Epe) with a total of 57 Local Council Development Authorities (LCDAs). From each of the administrative divisions, two LCDAs were randomly selected. From each of the 10 selected LCDAs, enumeration areas (EAs) were determined according to the National Population Commission (NPC) demarcation. All selected EAs were visited by research interviewers prior to the interview phase of the survey, and an enumeration and listing of all the household units contained therein were done. These lists were entered into a centralised computer data file, thus creating a sample in which the probability of any individual household being selected to participate in the survey was equal for every household within an EA. From the Kish formulae, the total number of households needed for representativeness was 5720 households. This was obtained by systematic random sampling of the households in each of the selected EAs.

\section{Ethical considerations}

The International Guidelines for Ethical Review of Epidemiological Studies was followed throughout the project. Considerations regarding confidentiality and the protection of research participants from harm, invasion of privacy, and the provision of emotional and practical support were given priority. The Ethics and Research Committee of the Lagos State University Teaching Hospital gave the ethical approval for the project. Written informed consent was obtained from the participants before the questionnaire was administered.

\section{Recruitment}

In the final stage of the selection, interviewers obtained a full listing of all residents above 18 years in each household, and from the list, they randomly selected at least two participants for the survey.

\section{Instruments}

The LSMHS instruments were included in a booklet used for each participant. The household questionnaire included the following:

1) Socio-demographic details: These included age in years, gender, occupation, working status, marital status, ethnicity, highest education and religion.

2) Assessment of common mental disorders: These were assessed using the relevant Patient Health Questionnaire (PHQ) modules. For example,

a Depression: Using the Depression module of PHQ (PHQ-9). ${ }^{11}$ This is a validated scale in Nigeria with a score of 10 and above considered for major depressive disorder (MDD). It had been validated in Nigeria with good psychometric properties. ${ }^{12}$

b Anxiety: Using the Anxiety module of PHQ (PHQ-7). ${ }^{13}$ This is also validated with a score of 10 and above considered for moderate to severe anxiety.

c Somatic symptoms: Using the Somatic module of PHQ (PHQ-15). ${ }^{14} \mathrm{~A}$ score of 10 and above is considered as medium to high somatic symptoms.
3) Assessment of alcohol use disorders: This was assessed using the short form of the Alcohol Use Disorders Identification Test (AUDIT-C). ${ }^{15}$ This well-validated scale has three items with a score of four and above for men and three and above for women considered as alcohol use disorders (AUDs).

4) Assessment of psychoactive substance use disorders: This was assessed using the modified Psychoactive Substance Use Disorders module of the Mini International Neuropsychiatric Interview (MINI). ${ }^{16}$ It was modified to include commonly used local substances. The DSM-IV criteria of endorsement of three or more of the items as substance use disorders (SUDs) were adhered to.

5) Assessment of disability: This was assessed using the 12-item version of the WHO Disability Assessment Schedule (WHODAS 2.0). ${ }^{17}$ A score of 13 and more on WHODAS is considered as moderate to severe disability.

6) Suicidal ideation: This was taken from Question 9 of the PHQ-9 which states 'Over the past 2 weeks, how often have you been bothered with the thought that you would be better off dead or of hurting yourself in some way'. The options include $0=$ not at all, $1=$ several days, $2=$ more than half the days and $3=$ nearly every day. Endorsing any of the options apart from 'not at all' is considered as having suicidal ideation according to the developers of the questionnaire.

All the instruments had been either well used or validated in Nigeria. All the instruments were translated into the three major languages and Pidgin English (spoken by nearly $80 \%$ of Lagos population) using the WHO translation protocols. The translated versions were pilot tested before data collection.

\section{Pre-fieldwork training and pilot study}

Research assistants $(n=50)$, supervisors $(n=5)$, monitors $(n=3)$ and the overall coordinator $(n=1)$ who were experienced field workers of the NPC with at least a university degree underwent a 1-week standardised training in interviewing skills and in administration of the questionnaire. Training includes field practical and dummy exercises. A pilot study was done to familiarise with the project sites. Each team established rapport with the community and opinion leaders in their respective areas, and discussed logistical issues including information dissemination, security for interviewers and privacy of the interview. During the household mapping and listing, posters and pamphlets on the project were distributed to each household. Announcements were made on the local radio and newspapers.

\section{Procedure and quality control}

Survey data collection was done over a period of 4 weeks of August to September 2015. Data collection was via face-to-face interviews using the paper and pencil method. In order to increase selfdisclosure, self-completion of the questionnaire booklet was encouraged for literate participants, and they were given the selfadministered booklets (of any language of their choice). For the non-literates, the questionnaires are read to them in private and their responses ticked. Data were collected on weekends (Saturday and Sunday) in order to maximise response. The five field supervisors were in each of one cluster, and they supervised the fieldwork, carried out daily field editing and ensured that reliable and quality data were obtained from the field. The three field monitors were on hand to ensure quality control. They functioned to spot-check the questions and get assurance from the supervisors that there is maximum cooperation on the field. When necessary they liaised with community leaders especially in the rural communities. All through the period, the project team was meeting with the data collection team to review the progress of the data collection. 


\section{Data analysis}

Data were centrally analysed using the IBM SPSS Statistics for Windows, Version $22.0^{18}$ by independent statisticians not working on the project. Weighting was applied to adjust for differences in sample according to the Nigerian population. Cross-tabulations were used to estimate current prevalence of suicidal ideation. Continuous variables and scores on the scales were divided into categories to minimise effects of extreme values. Logistic regression analysis modelling was used to analyse for factors associated with suicidal ideation. Logistic regression coefficients were converted to odds ratio and $95 \%$ confidence interval (95\% CI) for ease of interpretation. Statistical significance was set at 0.05 .

\section{Results}

\section{Socio-demographic profiles}

Out of the 11414 participants listed for interview, 168 could not be interviewed despite repeated home visits, so a total sample of 11246 were used making a response rate of $98.5 \%$. The largest number of participants $(n=3989,35.5 \%)$ was obtained from Ikeja division, followed by 3280 (29.1\%) from Ikorodu division, 1508 (13.4\%) from Badagry division, 1369 (12.2\%) from Lagos division and 1100 (9.8\%) from Epe division. The mean age was 36.75 (s.d.=12.3) years with a range of $18-74$ years. Age was further categorised with group 25-54 years accounting for $73.1 \%$ of the population. There were 6525 (58.0\%) females, 7797 (69.3\%) were married, 5958 (53.0\%) had secondary school education, 7526 (66.9\%) were petty traders or artisans, 2867 (25.5\%) were not presently working, 5503 (48.9\%) were from the Yoruba ethic group and 6276 (55.8\%) were Muslims. The rest of the socio-demographic profile is shown in Table 1.

\section{Mental health and disability profiles}

Table 2 shows that the mean score on PHQ-9 was 2.29 (s.d.=3.64) with 627 (5.6\%) having MDD. The mean score on PHQ-7 was 2.63 (s.d.=3.01) with 387 (3.4\%) having moderate to severe anxiety. The mean score on PHQ-15 was 3.58 (s.d.=3.43) with $712(6.3 \%)$ having medium to high somatic symptoms. The mean score on AUDIT-C was 0.93 (s.d.=1.94) with $976(8.7 \%)$ having AUDs, whereas the MINI was able to identify $237(2.1 \%)$ as having SUDs. The mean score on WHODAS-II was 4.45 (s.d. $=6.94)$ with $1239(11.0 \%)$ categorised as having moderate to severe disability.

\section{Rate of suicidal ideation}

Eight hundred and forty-six participants (7.5\%) endorsed having suicidal ideation in the past 2 weeks. These consisted of $667(6.0 \%)$ participants endorsing 'several days', 151 (1.3\%) endorsing 'more than half the days' and $28(0.2 \%)$ endorsing 'nearly every day'. Weighting was done with adjustment according to the Nigerian population statistics with the weighted prevalence of $7.28 \%$ (s. e. $=0.27)$.

\section{Associated factors}

A hierarchical logistic regression was used for the statistical analysis with the division or cluster entered first, followed by all socio-demographic variables. Because of the close association between depression and suicide, depression (MDD) was entered separately as the third step, followed by other common mental disorders of anxiety (moderate to severe anxiety), somatic symptoms (medium to high somatic symptoms), alcohol (AUDs) and substance use (SUDs) and lastly disability (moderate to severe disability). Table 3 shows the results of the regression analysis. The socio-demographic variables associated with suicidal ideation

\begin{tabular}{|c|c|c|}
\hline & Total & $\%$ \\
\hline \multicolumn{3}{|l|}{ Administrative divisions } \\
\hline Ikeja & 3989 & 35.5 \\
\hline Ikorodu & 3280 & 29.1 \\
\hline Badagry & 1508 & 13.4 \\
\hline Lagos Island & 1369 & 12.2 \\
\hline Epe & 1100 & 9.8 \\
\hline \multicolumn{3}{|l|}{ Age, years } \\
\hline $18-24$ & 1758 & 15.6 \\
\hline $25-54$ & 8220 & 73.1 \\
\hline $55-64$ & 895 & 8.0 \\
\hline$>64$ & 373 & 3.3 \\
\hline \multicolumn{3}{|l|}{ Gender } \\
\hline Male & 4721 & 42.0 \\
\hline Female & 6525 & 58.0 \\
\hline \multicolumn{3}{|l|}{ Marital status } \\
\hline Single & 2624 & 23.3 \\
\hline Married/cohabiting & 7797 & 69.3 \\
\hline Single/divorced/widowed & 825 & 7.3 \\
\hline \multicolumn{3}{|l|}{ Highest education level } \\
\hline No education & 575 & 5.1 \\
\hline Primary & 1884 & 16.8 \\
\hline Secondary & 5958 & 53.0 \\
\hline Tertiary & 2829 & 25.2 \\
\hline \multicolumn{3}{|l|}{ Occupation } \\
\hline Housewife/student/retiree & 2018 & 17.9 \\
\hline Petty trader/artisan & 7526 & 66.9 \\
\hline Civil servant & 771 & 6.9 \\
\hline Professionals/business & 931 & 8.3 \\
\hline \multicolumn{3}{|l|}{ Working status } \\
\hline Presently working & 8379 & 74.5 \\
\hline Presently not working & 2867 & 25.5 \\
\hline \multicolumn{3}{|l|}{ Ethnicity } \\
\hline Yoruba & 5503 & 48.9 \\
\hline Igbo & 2214 & 19.7 \\
\hline Hausa & 1822 & 16.2 \\
\hline Others & 1707 & 15.2 \\
\hline \multicolumn{3}{|l|}{ Religion } \\
\hline Christianity & 6276 & 55.8 \\
\hline Islam & 4490 & 39.9 \\
\hline Others & 480 & 4.3 \\
\hline
\end{tabular}

were older age (OR 1.65, 95\% CI 1.25-2.17 for age group 25-54 years and OR $2.13,95 \%$ CI $1.55-2.94$ for age group $>54$ years), female gender (OR 1.44, 95\%CI 1.22-1.72), not married (OR 1.35, 95\%CI 1.12-1.62) and low occupational group (OR 2.16, 95\% CI $1.62-2.87)$. Although all the common mental disorders of depression (OR 14.8, 95\% CI 11.65-18.00), anxiety (OR 1.71, 95\%CI 1.26-2.32), somatic symptoms (OR 2.14, 95\%CI 1.69-2.72) and disability (OR 1.36, 95\%CI 1.10-1.68) were significantly associated, AUD and SUD were not associated with suicidal ideation.

\section{Discussion}

\section{Validity of our findings}

We had set out to estimate the current prevalence of suicidal ideation and examine the associated factors using the data from the LSMHS. Despite the availability of cross-national figures from the well-conducted World Mental Health Survey Initiative, we were convinced of the need to conduct a local study that will inform policy and services in our setting. We deemed our findings very valid and comparable to the WMHS as we conducted a faceto-face interview with a sizeable population and high response rate. We estimated for current prevalence (instead of lifetime and 
Table 2 The clinical characteristics of the population

\begin{tabular}{|lcccc}
\hline Clinical variables & Mean score (s.d.) & Median score (range) & \multicolumn{1}{c}{ Clinically significant diagnosis } & Number (\% out of 11246) \\
Depression (PHQ-9) & 2.29 (s.d. 3.64) & $0.0(0-27)$ & Major depressive depression & $627(5.6 \%)$ \\
Anxiety (PHQ-8) & 2.63 (s.d. 3.01) & $2.0(0-20)$ & Moderate to severe anxiety & $387(3.4 \%)$ \\
Somatic symptoms (PHQ-15) & 3.58 (s.d. 3.43) & $3.0(0-26)$ & Medium to high somatic symptoms & $712(6.3 \%)$ \\
Alcohol use (AUDIT-C) & 0.93 (s.d. 1.94) & $0.0(0-11)$ & Alcohol use disorders & $976(8.7 \%)$ \\
Substance use (MINI) & & & Substance use disorders & $237(2.1 \%)$ \\
Disability (WHODAS 2.0) & 4.45 (s.d. 6.54) & $1.0(0-45)$ & Moderate to severe disability & $1239(11.0 \%)$ \\
\hline
\end{tabular}

12-months which are open to recall bias), used well-validated scales and included other variables peculiar to our local environment like somatic symptoms and disability assessment.

\section{Rate of suicidal ideation}

We found a weighted current prevalence of $7.28 \%$ for suicidal ideation from our study. Although this is less than the crossnational lifetime prevalence of $9.2 \%$ (s.e. $=0.1$ ) in the WMHS, ${ }^{8}$ it is far more than the reported Nigerian lifetime prevalence of $3.2 \%$ (s.e. $=0.2)$ in the same survey. ${ }^{8}$ These differences may be accounted for by the instruments used to score suicidal ideation or because of the different periods captured by the prevalence. We believed that a current prevalence is a better determinant (than lifetime prevalence) of the proportion of the population having suicidal ideation that may progress to planning and attempt. In low- and middle-income countries (LMICs) where resources are scarce, planning preventive services for suicide will require an accurate determination of the population to be catered for. We believe that our result is representative of the rest of the country, in that Lagos State is quite diverse in having all the over 250 tribes represented with the five administrative divisions having both rural and urban settings and vast differences in their socio-economic standards.
Although economically well developed, the living standards of the average Lagos resident are still considered far below what is obtainable in the Western culture. ${ }^{10}$

\section{Variables associated with suicidal ideation}

We found independent associations between suicidal ideation and socio-demographic factors (older age, female gender, not married and low occupational group), common mental disorders (depression, anxiety and somatic symptoms) and disability. All had modest magnitude (OR 1.35-2.16) except depression with a large magnitude (OR 14.48)

Contrary to the findings of the $\mathrm{WMHS}^{8}$ of an association between younger age and suicidal ideation, we found increasing age group to be significantly associated with suicidal ideation. This is quite significant as our finding is in direct correlation with the crude-age-specific estimated suicide rates for Nigeria in the WHO Global Health Estimates. ${ }^{2}$ Although our study did not evaluate suicidal plans and attempt, we believe our finding deserves more investigations as the WMHS also found that transition from suicidal ideation to first onset of plan or attempt is extremely elevated within the first one year of the ideation and decreases substantially thereafter. This raises the question if assessing 'lifetime' prevalence

Table3 Regression analysis table showing odds ratio (OR) and $95 \%$ confidence interval $(95 \% \mathrm{Cl})$ for factors significantly associated with suicidal ideation

\begin{tabular}{|c|c|c|c|c|}
\hline Variables & $\%$ & $\mathrm{OR}(95 \mathrm{Cl})$ & Wald & $P$ \\
\hline \multicolumn{5}{|l|}{ Socio-demographic } \\
\hline \multicolumn{5}{|l|}{ Age } \\
\hline 18-24 years & 5.6 & 1 (reference) & & \\
\hline $25-54$ years & 7.4 & $1.65(1.25-2.17)$ & 12.36 & $<0.001$ \\
\hline 55 years and above & 10.7 & $2.13(1.55-2.944)$ & 21.47 & $<0.001$ \\
\hline \multicolumn{5}{|l|}{ Gender } \\
\hline Male & 6.0 & 1 (reference) & & \\
\hline Female & 8.6 & $1.44(1.22-1.72)$ & 18.23 & $<0.001$ \\
\hline \multicolumn{5}{|l|}{ Marital status } \\
\hline Married & 7.1 & 1 (reference) & & \\
\hline Not married ${ }^{a}$ & 8.5 & $1.35(1.12-1.62)$ & 10.32 & 0.001 \\
\hline \multicolumn{5}{|l|}{ Occupational status ${ }^{\mathrm{b}}$} \\
\hline High level & 3.8 & 1 (reference) & & \\
\hline Low level & 8.2 & $2.16(1.62-2.87)$ & 27.88 & $<0.001$ \\
\hline \multicolumn{5}{|l|}{ Mental disorders } \\
\hline \multicolumn{5}{|l|}{ Depression } \\
\hline No/mild depression & 4.9 & 1 (reference) & & \\
\hline Major depressive disorder & 51.8 & $14.48(11.65-18.00)$ & 578.74 & $<0.001$ \\
\hline \multicolumn{5}{|l|}{ Anxiety } \\
\hline No/mild anxiety & 6.9 & 1 (reference) & & \\
\hline Moderate to severe anxiety & 24.5 & $1.71(1.26-2.32)$ & 11.86 & $<0.001$ \\
\hline \multicolumn{5}{|l|}{ Somatic symptoms } \\
\hline No/low somatic symptoms & 5.8 & 1 (reference) & & \\
\hline Medium to high somatic symptoms & 32.3 & $2.14(1.69-2.72)$ & 39.58 & $<0.001$ \\
\hline \multicolumn{5}{|l|}{ Disability } \\
\hline No/mild disability & 6.0 & 1 (reference) & & \\
\hline Moderate to severe disability & 19.5 & $1.36(1.10-1.68)$ & 8.21 & 0.004 \\
\hline
\end{tabular}


for suicidal ideation may be biased because of forgetting and recallbias as the person ages. It must be stated, however, that suicide may be highly represented in subjects who answered 'not at all' and showed no suicidal ideation, so suicide prevention programmes should not only rely on suicidal ideation for projection. As expected and like the findings of the cross-national WMHS and other surveys, we found female gender and not being married to be significantly associated with suicidal ideation. We also found suicidal ideation to be higher among people within the lower occupational group.

Like earlier surveys, we found common mental disorders to be significantly related to suicidal ideation. This relationship was of very high magnitude with depression. Although it is in line with the WHMS report (Nigeria being one of the seven countries with ORs significantly higher than the cross-national estimates), it may also be partly because of our assessment of suicidal ideation using an item from the overall assessment of depression. It should be noted that the WMHS report that impulse control disorder was a stronger predictor of suicidal ideation in LMICs did not include data from Nigeria. In our study, we found somatic symptoms (a common finding in LMICs) to be significantly associated with suicidal ideation. One interesting finding in our study was that both AUD and SUD dropped out of our regression analysis and were not predictive of suicidal ideation. It will be interesting to assess if they rather predict suicidal attempt or plan in those with ideation. Unique to our study was the association of disability with suicidal ideation; this warrants further investigation as there is well-established relationship between disabling chronic disorders, mental illness and suicide.

\section{Implications of our study}

Our study suggests that despite the success and validity of large cross-national surveys, individual countries, states and districts may still need to generate complementary local data to plan for suicide prevention and develop timely and effective response. We have validated the earlier findings of mental disorders being the main factors associated with suicidal ideation, but our study emphasises the need for further research that will not only explain the variability in the rate, but have local factors specific to the country as part of the model for risk factors.

\section{Limitations and strengths}

First, we had used a single item on a depression rating scale to make a diagnosis of suicidal ideation; although this is a widely accepted item, it could have biased the result and not provided a very valid and detailed report on suicidal ideation. Second, we measured only common mental disorders using rating scales, although these scales are well validated in this environment with good psychometric properties. Thirdly, we assessed only for suicidal ideation and were not able to predict how ideation may lead to plan or attempt. Although our study was in Lagos State, it can be said to be representative of the general situation in Nigeria as Lagos is cosmopolitan in nature encompassing all the tribes and religious groups in the country and is about $10 \%$ of the country's population. The strength of our study lies in its assessing for the current prevalence of suicidal ideation, including locally relevant variables like somatic symptoms and disability and our moderately large sample size.

Despite the validity of cross-national surveys, there is need for individual countries to generate complementary local data to explain variability in rates and risk factors in order to plan for suicide prevention or develop timely and effective response.
Abiodun 0. Adewuya, MPH, FWACP, FMCPscyh, Department of Behavioura Medicine, Lagos State University College of Medicine, Lagos, Nigeria; Centre for Menta Health Research \& Initiative, Lagos, Nigeria; Bolanle A. Ola, FWACP, FMCPsych, PhD, Department of Behavioural Medicine, Lagos State University College of Medicine, Lagos, Nigeria; Olurotimi A. Coker, MSC, FWACP, Department of Behavioural Medicine, Lagos State University College of Medicine, Lagos, Nigeria; Olayinka Atilola FWACP, FMCPSych, Department of Behavioural Medicine, Lagos State University College of Medicine, Lagos, Nigeria; Mathew P. Zachariah, PhD, Department of Behavioural Medicine, Lagos State University College of Medicine, Lagos, Nigeria; Olufemi Olugbile, FMCPsych, FRCPsych, Synthesiz Consults, Lagos, Nigeria; Adedolapo Fasawe, MBChB, MPH, Lagos State Ministry of Health, Lagos, Nigeria; Olajide Idris, MBBS, MPH, Lagos State Ministry of Health, Lagos, Nigeria

Correspondence: Abiodun O. Adewuya, Department of Behavioural Medicine, Lagos State University College of Medicine, Lagos, Nigeria. E-mail: Abiodun.adewuya@ lasucom.edu.ng

First received 22 Nov 2016, final revision 22 Nov 2016, accepted 23 Nov 2016

\section{Funding}

The Lagos State Mental Health Survey (LSMHS) Project was funded by a grant from the Lagos State Ministry of Health (LSMOH)

\section{References}

1 World Health Organization. WHO Mortality Database Documentation: 1 May 2013 Update. WHO, 2013

2 World Health Organization. Prevention of Suicide: Guidelines for the Formulation and Implementation of National Strategies. WHO, 1996.

3 Vijayakumar L, Nagaraj K, Pirkis J, Whiteford H. Suicide in developing countries 1: frequency, distribution, and association with socioeconomic indicators. Crisis 2005; 26: 104-11.

4 World Health Organization. mhGAP Intervention Guide for Mental, Neurological and Substance Use Disorders in Non-specialized Health Settings. WHO, 2010.

5 World Health Organization. Mental Health Action Plan 2013-2020. WHO, 2013

6 Robins LN, Regier DA (eds). Psychiatric Disorders in America: The Epidemiologic Catchment Area Study. The Free Press, 1991.

7 Kessler RC, McGonagle KA, Zhao S, Nelson CB, Hughes M, Eshleman S, et al. Lifetime and 12-month prevalence of DSM-III-R psychiatric disorders in the United States. Results from the National Comorbidity Survey. Arch Gen Psychiatry 1994; 51: 8-19.

8 Nock MK, Borges G, Bromet EJ, Alonso J, Angermeyer M, Beautrais A, et al. Crossnational prevalence and risk factors for suicidal ideation, plans and attempts. $\mathrm{Br} J$ Psychiatry 2008; 192: 98-105.

9 Lagos State Government. Population Census of Lagos State. LASG Press, 2016.

10 World Population Review. Lagos Population 2016 (26 October; http://worldpopulation review.com/world-cities/lagos-population/).

11 Kroenke K, Spitzer RL, Williams JB. The PHQ-9: validity of a brief depression severity measure. J Gen Int Med 2001; 16: 606-13.

12 Adewuya AO, Ola BA, Afolabi OO. Validity of the Patient Health Questionnaire (PHQ-9) as a screening tool for depression amongst Nigerian university students. J Affect Disord 2006; 96: 89-93.

13 Spitzer RL, Kroenke K, Williams JBW, Löwe B. A brief measure for assessing generalized anxiety disorder: the GAD-7. Arch Intern Med 2006; 166: 1092-7.

14 Kroenke K, Spitzer RL, Williams JBW. The PHQ-15: validity of a new measure for evaluating somatic symptom severity. Psychosom Med 2002; 64: 258-66.

15 Bush K, Kivlahan DR, McDonell MB, Fihn SD, Bradley KA. The AUDIT alcohol consumption questions (AUDIT-C): an effective brief screening test for problem drinking. Ambulatory Care Quality Improvement Project (ACQUIP). Alcohol Use Disorders Identification Test. Arch Intern Med 1998; 158: 1789-95.

16 Sheehan D, Lecruiber Y, Sheehan $\mathrm{KH}$, Amorim P, Janavs J, Weiller E, et al. The Mini International Neuropsychiatric Interview (M.I.N.I.): The development and validation of a structured diagnostic psychiatric interview. J Clin Psychiatry 1998; 59: 22-33.

17 Üstün TB, Kostanjsek N, Chatterji S, Rehm J. Measuring Health and Disability: Manual for WHO Disability Assessment Schedule (WHODAS 2.0). WHO, 2010.

18 IBM Corp. IBM SPSS Statistics for Windows, Version 22.0. IBM Corp, 2013. 